\title{
A SHORT NOTE ON NESTED SUMS
}

\author{
NIR SHVALB AND SHLOMI HACOHEN
}

Received 11 April, 2016

\begin{abstract}
We express a set of nested sums as a 1-index weighted sum using Stars and Bars The-
\end{abstract} orem.

2010 Mathematics Subject Classification: 05A17; 11P21

Keywords: nested sum, Frobenius equation, stars and bars

\section{INTRODUCTION}

Nested sums appear in various fields of research. Here, we consider the following summation:

$$
\sum_{a_{1}=0}^{N} \sum_{a_{2}=0}^{a_{1}} \sum_{a_{3}=0}^{a_{2}} \cdots \sum_{a_{n}=0}^{a_{n-1}} f\left(\alpha_{1} a_{1}+\alpha_{2} a_{2}+\cdots+\alpha_{n} a_{n}\right)
$$

where $f(m)$ may be any map defined for $0 \leq m \leq 2 N$. Under certain constraints on the coefficients $\alpha_{i}, i \in\{1, \ldots, n\}$, we simplify the expression to the form $\sum_{\gamma} f(\gamma) P(\gamma)$ where $P(\gamma)$ is the number of occurrences of $\gamma$ within the sum. Surprisingly, the authors could not find any formula concerning such expressions. Consider for example the sum:

$$
S=\sum_{a_{1}=0}^{N} \sum_{a_{2}=0}^{a_{1}} \sum_{a_{3}=0}^{a_{2}} \sum_{a_{4}=0}^{a_{3}} f\left(2 a_{3}-a_{4}\right),
$$

applying Theorem 1 below, simplifies the expression to:

$$
S=\sum_{\gamma=0}^{2 N} \sum_{\phi=\phi_{\min }(\gamma)}^{\phi_{\max }(\gamma)}\left(\begin{array}{c}
N-\gamma+\phi+2 \\
2
\end{array}\right) f(\gamma)
$$

where, $\phi_{\min }(\gamma)=\max \{0, \gamma-N\}$ and $\phi_{\max }(\gamma)=\min \{N,\lfloor\gamma / 2\rfloor\}$.

(c) 2018 Miskolc University Press 


\section{DISCUSSION}

Denote $\underline{\alpha}=\left(\alpha_{1}, \ldots, \alpha_{n}\right)^{T}$ and $\underline{a}=\left(a_{1}, \ldots, a_{n}\right)$ and $\gamma=\underline{\alpha} \cdot \underline{a}$ we set:

$$
\begin{aligned}
x_{1} & =N-a_{1}, \\
x_{2} & =a_{1}-a_{2}, \\
& \vdots \\
x_{n} & =a_{n-1}-a_{n}, \\
x_{n+1} & =a_{n}
\end{aligned}
$$

so

$$
N=x_{1}+x_{2}+\cdots+x_{n+1}
$$

where $0 \leq x_{i} \leq N, i \in\{1, \ldots, n+1\}$. This implies that:

$$
\gamma=N \beta_{1}-x_{1} \beta_{1}-x_{2} \beta_{2} \cdots-x_{n} \beta_{n}
$$

where $\beta_{j}=\sum_{i=j}^{n} \alpha_{i}$.

Substituting into Eq. 2.1 we get the Frobenius Equations (cf. [2]):

$$
N \beta_{1}-\gamma=x_{1} \beta_{1}+\ldots+x_{n} \beta_{n}
$$

The number of integer solutions to this equation yields the following:

Theorem 1. Consider the sum:

$$
S=\sum_{a_{1}=0}^{N} \sum_{a_{2}=0}^{a_{1}} \sum_{a_{3}=0}^{a_{2}} \cdots \sum_{a_{n}=0}^{a_{n-1}} f\left(\alpha_{1} a_{1}+\alpha_{2} a_{2}+\cdots+\alpha_{n} a_{n}\right)
$$

Denote by $d^{+}$the set of indices of coefficients $\beta_{j}=\sum_{i=j}^{n} \alpha_{i}$ that are equal to +1 , by $d^{0}$ the set of coefficients' indices that vanish and by $d^{-}$the set of coefficients' indices that are equal -1 . Moreover we assume that $\beta_{1}=1$. Then

$$
S=\sum_{\gamma=0}^{2 N} P(\gamma, \underline{\alpha}, N) f(\gamma)
$$

where the occurrence function is:

$$
P(\gamma, \underline{\alpha}, N)= \begin{cases}\left(\begin{array}{c}
N-\gamma+\left\|\mathcal{I}^{+}\right\|-1 \\
\left\|\mathcal{l}^{+}\right\|-1
\end{array}\right) & \text { if }\left\|\mathcal{l}^{-}\right\|=0 \\
\sum_{\varphi=\varphi_{\min }(\gamma)}^{\varphi_{\max }(\gamma)}\left(\begin{array}{c}
\varphi+\left\|\mathcal{L}^{-}\right\|-1 \\
\left\|\mathcal{L}^{-}\right\|-1
\end{array}\right)\left(\begin{array}{c}
N-\gamma+\varphi+\left\|\mathcal{L}^{+}\right\|-1 \\
\left\|\mathcal{L}^{+}\right\|-1
\end{array}\right) & \text { otherwise }\end{cases}
$$


and

$$
\varphi_{\min }(\gamma)=\max \{0, \gamma-N\}, \quad \varphi_{\max }(\gamma)=\min \left\{N\left\|\mathcal{d}^{-}\right\|,\left\lfloor\frac{\gamma}{2}\right\rfloor\right\} .
$$

Proof. Recall the "Stars and Bars Theorem" by W. Feller [1]: "For any pair of positive integers $n$ and $k$, the number of $k$-tuples of non-negative integers whose sum is $n$ is equal to $\left(\begin{array}{c}n+k-1 \\ k-1\end{array}\right)$ ".

So in the case where $\left\|d^{-}\right\|=0$, the number of solutions for Eq. 2.2 takes the desired form. (Here \|\| denotes the cardinality of a set). In the general case we have $\left\|\mathscr{d}^{+}\right\|,\left\|\mathcal{d}^{-}\right\|,\left\|\mathcal{d}^{0}\right\|>0$, and Eq. 2.2 takes the form:

$$
N-\gamma+\sum_{k \in \mathcal{I}^{-}} x_{k}=\sum_{i \in \mathcal{l}^{+}} x_{i}
$$

Equation $\varphi=\sum_{k \in \mathcal{I}^{-}} x_{k}$ have $\left(\begin{array}{c}\varphi+\left\|\mathcal{l}^{-}\right\|-1 \\ \left\|\mathcal{l}^{-}\right\|-1\end{array}\right)$ roots. The number of solutions to the equation $N-\gamma+\varphi=\sum_{i \in \mathcal{I}^{+}} x_{i}$ (thinking of the left hand side as a constant value) is $\left(\begin{array}{c}N-\gamma+\varphi+\left\|\ell^{+}\right\|-1 \\ \left\|\ell^{+}\right\|-1\end{array}\right)$. Summing over $\varphi$ yields:

$$
P(\gamma, \underline{\alpha}, N)=\sum_{\varphi=\varphi_{\min }}^{\varphi_{\max }}\left(\begin{array}{c}
\varphi+\left\|\mathcal{d}^{-}\right\|-1 \\
\left\|\mathcal{l}^{-}\right\|-1
\end{array}\right)\left(\begin{array}{c}
N-\gamma+\varphi+\left\|\mathcal{d}^{+}\right\|-1 \\
\left\|\mathcal{d}^{+}\right\|-1
\end{array}\right)
$$

To produce the upper and lower bound of $\varphi$ for the summation above note that $N \geq$ $x_{i} \geq 0$ for all $x_{i}$ 's so $N\left\|d^{-}\right\| \geq \varphi \geq 0$. Eq. 2.3 along with the fact that $\beta_{1}=1$ (since it must be positive by definition) then implies $\varphi \leq N\left(\left\|\alpha^{+}\right\|-1\right)+\gamma$. Substituting Eq. 2.1 into Eq. 2.2 yields:

$$
\begin{aligned}
\gamma=\left(\beta_{1}-\beta_{2}\right) x_{2}+\left(\beta_{1}-\beta_{3}\right) x_{3}+\cdots+ & \left(\beta_{1}-\beta_{n}\right) x_{n}+\beta_{1} x_{n+1} \\
& =2 \sum_{k \in \ell^{-}} x_{k}+\sum_{k \in l^{-}} x_{k} x_{j}+x_{n+1}
\end{aligned}
$$

where we used:

$$
\beta_{1}-\beta_{i}= \begin{cases}0 & i \in d^{+} \\ 1 & i \in d^{0} \\ 2 & i \in d^{-}\end{cases}
$$

Note that the maximal $\gamma$ occurs when $\sum_{k \in \mathcal{l}^{-}} x_{k}=N$ so that $\sum_{k \in \mathcal{I}^{-}} x_{k}=0$ (see 2.1). Taking the two last terms of Eq. 2.5 to zero, it yields $\varphi \leq \frac{\gamma}{2}$. Since $\left(\begin{array}{l}a \\ b\end{array}\right)$ vanishes when $b>a$ we also require $\varphi \geq \gamma-N$. Finally, we obtain:

$$
\varphi_{\min }(\gamma)=\max \{0, \gamma-N\}, \quad \varphi_{\max }(\gamma)=\min \left\{N\left\|d^{-}\right\|,\left\lfloor\frac{\gamma}{2}\right\rfloor\right\}
$$


Example 1. Consider the case where $n$ is odd and $\left\|d^{-}\right\|=0$ (e.g. $\gamma$ is the alternating sum: $a_{1}-a_{2}+a_{3}-\cdots+a_{n}$. Let $f(k, n, p)$ be the probability mass binomial function $\left(\begin{array}{l}n \\ k\end{array}\right) p^{k}(1-p)^{n-k}$. Applying Theorem 1 yields :

$$
\begin{aligned}
\sum_{a_{1}=0}^{N} \sum_{a_{2}=0}^{a_{1}} \ldots \sum_{a_{n}=0}^{a_{n-1}} f\left(\left\|d^{+}\right\|,\right. & N-\gamma, p) \\
& =\frac{p^{\left\|d^{+}\right\|-1}}{\left(\left\|d^{+}\right\| !\right)^{2}}\left[(1-p)^{-N-\left\|d^{+}\right\|-1}-(1-p)^{N-\left\|d^{+}\right\|}\right]
\end{aligned}
$$

where we used the identity $\left(\begin{array}{c}a-b \\ b\end{array}\right)\left(\begin{array}{l}a \\ b\end{array}\right)=(b !)^{-2}$.

\section{REFERENCES}

[1] W. Feller, An Introduction to Probability Theory and Its Applications. Wiley, Vol 1, 2nd ed., 1950.

[2] S. Sertöz, "On the number of solutions of a Diophantine equation of Frobenius," Discrete Mathematics and Applications, vol. 8, pp. 153-162, 1998, doi: 10.1515/dma.1998.8.2.153.

\section{Authors' addresses}

\section{Nir Shvalb}

Ariel University, Faculty of Engineering, Ariel, 40700, Ariel, Israel

E-mail address: nirsheariel.ac.il

\section{Shlomi Hacohen}

Ariel University, Faculty of Engineering, Ariel, 40700, Ariel, Israel

E-mail address: hacohenshlomiegmail.com 